Check for updates

Cite this: RSC Adv., 2017, 7, 42900

\title{
Glucuronoxylan-mediated silver nanoparticles: green synthesis, antimicrobial and wound healing applications
}

\author{
Gulzar Muhammad, ${ }^{a}$ Muhammad Ajaz Hussain, (D) *a Muhammad Amin, ${ }^{a}$ \\ Syed Zajif Hussain, ${ }^{\mathrm{b}}$ Irshad Hussain, (D) ${ }^{\mathrm{b}}$ Syed Nasir Abbas Bukhari (D) \\ and Muhammad Naeem-ul-Hassan (iD ${ }^{a}$
}

Hydrogel forming polysaccharides are attracting attention for the design of diverse nature silver nanoparticles (Ag NPs) with potential biological applications. Herein, we report the green synthesis of Ag NPs and their antimicrobial activity along with their potential application in wound dressings. Glucuronoxylan (GX) isolated from seeds of Mimosa pudica (MP) was used as a stabilizing/capping agent to prepare the NPs under diffused sunlight using different concentrations of $\mathrm{AgNO}_{3}$. The progress of the reaction was monitored using a UV-visible spectrophotometer and characteristic surface plasmon absorption (SPR) bands were found in the range of 390-465 nm. The morphology and elemental composition of the synthesized Ag NPs were analyzed using scanning electron microscopy equipped with STEM and EDS detectors and the diameter of the Ag NPs was found to be in the range of 2 to $18 \mathrm{~nm}$. The synthesized NPs exhibited antimicrobial potential against Staphylococcus epidermidis, Escherichia coli, Pseudomonas aeruginosa, Bacillus subtilis, Staphylococcus aureus, Aspergillus niger, Penicillium notatum, Rhizopus stolonifer and Actinomycetes odontolyticus. The minimal inhibitory concentrations (MICs) of the Ag NPs against different pathogenic strains were determined. Moreover, the dressing prepared from Ag NP impregnated GX exhibited a remarkable wound healing potential in rabbits. In situ synthesized Ag NPs were stored in the form of GX thin films and their UV/vis spectra exhibited that the texture of the Ag NPs remained intact after a six month storage period.

Received 9th July 2017

Accepted 31st August 2017

DOI: $10.1039 / c 7 r a 07555 c$

rsc.li/rsc-advances

\section{Introduction}

Nanotechnology is emerging rapidly nowadays because of the amazing optical, magnetic, catalytic, electrical and biological properties of NPs as compared to their bulk states. ${ }^{1}$ Size, shape and surface chemistry are important factors in controlling the aforementioned characteristics of NPs. ${ }^{2} \mathrm{Ag}$ NPs are commonly synthesized (320 tons per year) due to their microbicidal nature. ${ }^{3}$ In addition to this, Ag NPs also find applications in bioimaging, sensing, cosmetics, textiles, household foodstuffs, room sprays and tumor treatments. ${ }^{4-6}$

The synthesis of Ag NPs can be achieved using different toxic reducing agents and solvents as well as non-biodegradable complexing and stabilizing agents. ${ }^{7-9}$ Moreover, for biomedical applications, $\mathrm{Ag}$ NPs are preferably synthesized using green approaches ${ }^{\mathbf{1 0 1 1}}$ such as microemulsion, ${ }^{12} \gamma$-rays, ${ }^{13}$ diffused

${ }^{a}$ Department of Chemistry, University of Sargodha, Sargodha 40100, Pakistan. E-mail: majaz172@yahoo.com

${ }^{b}$ Department of Chemistry, SBA School of Science \& Engineering (SSE), Lahore University of Management Sciences (LUMS), DHA, Lahore Cantt 54792, Pakistan

${ }^{c}$ Department of Pharmaceutical Chemistry, College of Pharmacy, Al Jouf University, Sakaka, Al Jouf 2014, Kingdom of Saudi Arabia. E-mail: snab_hussaini@yahoo.com sunlight and UV-irradiation, ${ }^{\mathbf{1 4}}$ microwave irradiation $^{\mathbf{1 5}}$ and spray pyrolysis. ${ }^{\mathbf{1 6}}$

Researchers have explored polysaccharides (i.e., non-toxic, biodegradable and economical reagents) ${ }^{17,18}$ such as hydroxypropylcellulose, ${ }^{19}$ dextran, ${ }^{20}$ cellulose $^{21}$ and chitosan $^{22}$ as reducing and stabilizing agents for the green synthesis of $\mathrm{Ag}$ NPs. Among polysaccharides, naturally occurring water swellable polysaccharides like arabinoxylans ${ }^{23}$ and rhamnogalacturonan ${ }^{24}$ are being explored nowadays for green synthesis of silver nanoparticles (Ag NPs) having diverse morphology and potential biological applications.

Polysaccharide based hydrogels are smart materials widely used in cosmetics, pharmaceutical and daily life. ${ }^{25-27}$ Such hydrogels can be utilized as capping and reducing agent for the synthesis of Ag NPs with virtually no reagent. ${ }^{19,20,24}$ In this connection, glucuronoxylan (GX) (Fig. 1) is a hydrogel forming polysaccharide which could be a potential candidate. Recently, GX has been isolated from MP seeds and proved to be a potential carrier for targeted release of different drugs due to its $\mathrm{pH}$ responsive on-off switching. ${ }^{28-33}$ This versatile biomaterial GX has also been employed for the alleviated temperature assisted synthesis of Ag NPs. ${ }^{34}$ 


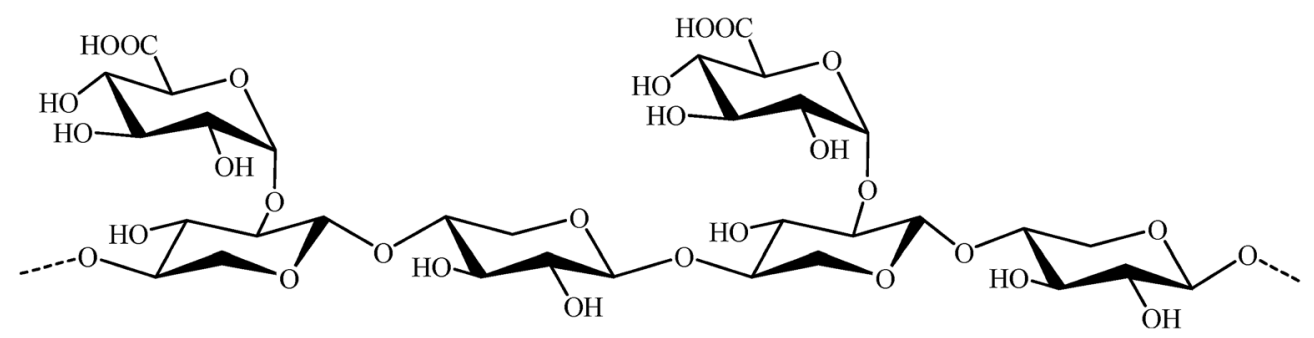

Fig. 1 The plausible structure of GX extruded from seed coat of MP.

Present work is focusing on diffused sunlight assisted green, easy and rapid synthesis of Ag NPs using GX, which is economical, non-toxic and eco-friendly method. Morphology and mechanism of formation of NPs are also discussed here. Aim is to explore the antibacterial and antifungal activities of GX protected Ag NPs (GX-Ag NPs) against strains like S. epidermidis (ATCC 12228), E. coli (ATCC 25922), P. aeruginosa (ATCC 27853), B. subtilis (ATCC 6633), S. aureus (ATCC 25923) and $A$. niger, $P$. notatum, $R$. stolonifer and $A$. odontolyticus, respectively. We are aimed to study wound healing application of as-synthesized GX-Ag NPs as well. As the GX has potential to form thin films on drying like other polysaccharides therefore potential of GX-based thin films as a storage medium for the $\mathrm{Ag}$ NPs is being studied.

\section{Materials and methods}

\subsection{Materials}

Seeds of MP were purchased from local market. After grading and manual cleaning, MP seeds were stored at room temperature. Hydrogel from seed coats of MP were extruded using deionized water. $\mathrm{AgNO}_{3}$ (99.98\%) from Merck, Darmstadt, Germany was used as a silver precursor. All the solution of $\mathrm{AgNO}_{3}$ and suspension of GX were prepared in deionized water. All reagents used were of analytical grade.

\subsection{Isolation of hydrogel}

MP cleaned seeds $(100 \mathrm{~g})$ were soaked in water $(1 \mathrm{~L})$ for $12 \mathrm{~h}$, and then warmed at $50{ }^{\circ} \mathrm{C}$ for $30 \mathrm{~min}$. The hydrogel extruded by seeds was separated using a cotton muslin cloth and then repeatedly washed with $n$-hexane to make it free from fat soluble substances. The separated hydrogel was dried at $50{ }^{\circ} \mathrm{C}$, ground to fine powder, passed through 60 mesh sieve and stored in a vacuum desiccator.

\subsection{Preparation of $\mathrm{AgNO}_{3}$ solution and GX hydrogel}

$\mathrm{AgNO}_{3}(0.85,1.27$ and $1.7 \mathrm{~g})$ was dissolved in deionized water $(100 \mathrm{~mL})$ to prepare 50,75 and $100 \mathrm{mmol}$ solutions, respectively. GX $(1.0 \mathrm{~g})$ was added into deionized water $(100 \mathrm{~mL})$ to obtain its hydrogel.

\subsection{Synthesis of GX mediated Ag NPs}

Freshly prepared GX hydrogel $(2 \mathrm{~mL})$ was homogenized with $\mathrm{AgNO}_{3}(50 \mathrm{mmol}, 2 \mathrm{~mL})$ solution in dark. After this, mixture was exposed to sunlight and progress of reaction was noted by change in colour and measuring characteristic surface plasmon resonance (SPR) absorption band using UV-vis spectrophotometer over a period of $24 \mathrm{~h}$. Using same procedure, colour changes were also observed for $\mathrm{AgNO}_{3}$ (75 and $100 \mathrm{mmol}$ ) solutions, respectively.

\section{$2.5 \quad \mathrm{UV} / \mathrm{vis}$ spectrophotometry}

Diffused sunlight assisted reduction of silver ions was monitored by recording the characteristic SPR absorption peaks of GX-Ag NPs at fixed intervals of time, i.e., $0.5,1,2,5$ and $24 \mathrm{~h}(8 \mathrm{~h}$ daily exposure to sunlight) in a wavelength ranging from 800 to $200 \mathrm{~nm}$ using UV/vis spectrophotometer (UV-1700, PharmaSpec, Shimadzu Corporation, Kyoto, Japan). Effect of concentration and time on the synthesis of Ag NPs was also studied.

\subsection{FTIR spectroscopy}

Fourier transform infrared (FTIR) spectra of GX with or without Ag NPs were recorded on an IR Prestige-21 (Shimadzu, Japan) spectrometer using the KBr pellet technique. The samples and pellets were desiccated under vacuum before examination.

\subsection{Scanning electron microscopy-energy dispersive X-ray spectroscopy (SEM-EDS)}

The surface morphology and elemental composition of GX-Ag NPs was studied by blotting their drop on to carbon coated copper grid using scanning electron microscope (FEI Nova, NanoSEM 450) equipped with transmission electron mode (STEM detector) and energy dispersive X-ray (EDX) detector operating at $10 \mathrm{kV}$ and working distance of $5 \mathrm{~mm}$.

\subsection{Film formation of GX embedded Ag NPs}

A mixture of $\mathrm{AgNO}_{3}(50 \mathrm{mmol})$ and $\mathrm{GX}$ was exposed to sunlight for $5 \mathrm{~h}$. Mixture was air dried ( 2 days) in dark at room temperature in a Petri dish to produce GX thin films embedded with Ag NPs and stored in a desiccator. After different time intervals, dry film was homogenized in deionized water, and the resultant suspension was characterized through UV/vis spectrophotometry after filtration (with $0.45 \mu$ syringe filter) and sonication. Furthermore, $\mathrm{Ag}$ NPs were isolated from this suspension after centrifugation and then characterized by powder X-ray diffraction (PXRD) as well. 


\subsection{Antimicrobial potential of GX-Ag NPs}

$\mathrm{Ag}$ NPs were investigated for their fungicidal and bactericidal effects against bacterial and fungal strains. The tested bacterial strains include $E$. coli American-type culture collection ATCC 25922, B. subtilis ATCC 6633, S. aureus ATCC 25923, P. aeruginosa ATCC 27853, S. epidermidis ATCC 12228 and A. odontolyticus ATCC 17929. Fungal strains used for antimicrobial assay were $P$. notatum, $R$. stolonifer and A. niger. Bacterial and fungal strains were grown on Mueller-Hinton agar media (Thermo Fisher Scientific, Waltham, MA, USA) and Sabouraud dextrose agar (Hardy Diagnostics, Santa Maria, CA, USA) respectively. Bacterial cultures were incubated for $24 \mathrm{~h}$ at $37^{\circ} \mathrm{C}$ and fungal cultures for $30-37 \mathrm{~h}$ at $27-30{ }^{\circ} \mathrm{C}$. After 7 days, fungal cultures were washed with deionized water and suspended in normal saline solution. Once again, fungal cultures were incubated at $28{ }^{\circ} \mathrm{C}$ after passing through aseptic glass wool. The growth of cultures was stimulated by shaking the cultures at times and 0.5 McFarland standards were followed to check the turbidity of the inoculums. For the evaluation of microbicidal effect of GX-Ag NPs, disc diffusion method was employed. ${ }^{35}$ Discs $(6 \mathrm{~mm})$ of filter paper (Whatman no. 1) were carefully loaded with NPs and put on microbial cultures in Petri plates. Incubation of bacterial and fungal strains was carried out respectively at $37^{\circ} \mathrm{C}$ for $24 \mathrm{~h}$ and $30{ }^{\circ} \mathrm{C}$ for $36 \mathrm{~h}$. Negative control used was the deionized water. All experiments were repeated thrice and average values were discussed.

To perform MIC and to get reasonable response a standard number of colony forming units (CFU) were maintained for different bacterial strain, ${ }^{36}$ though it is difficult to maintain the exact no of CFU for all strain. Because different bacterial species have different optical densities due to their variable size, cell wall texture and turbidity of growth medium. So for the certainty of uniform number of bacteria, a set of graph was prepared by studying the log and decline curve for each bacterial strain. Thus, a final concentration of $5 \times 10^{6} \mathrm{CFU} \mathrm{mL}^{-1}$ was measured. ${ }^{37,38}$ MIC was determined using serial dilutions of $\mathrm{Ag}$ NPs from 200 to $0.390 \mu \mathrm{g} \mathrm{mL}^{-1}$.

\subsection{Preparation of wound dressing}

GX-Ag NPs were examined for wound healing capability and dressing patch was designed with two layers, cubic porous adhesive (USP) backing membrane $(5 \times 5 \mathrm{~cm})$ and the cubic sterile cotton patch $(4 \times 4 \mathrm{~cm})$. The dressing patch was dipped into mixture of GX-Ag NPs $(50 \mathrm{mmol}, 5 \mathrm{~h}$ sunlight exposed sample) and placed on adhesive membrane as an inner wound dressing. A fine layer of GX hydrogel was applied with spatula on inner most layer of bandage.

\subsection{Wound healing studies}

EU directive ethical guidelines (2010/63) were followed while conducting experiments on animals. ${ }^{39}$ Three groups of healthy male albino rabbits $(2.2 \pm 0.2 \mathrm{~kg}, n=3)$ and Wistar rats $(350 \pm$ $20 \mathrm{~g}, n=6$ ) were selected for wound healing studies. Animals were fed with routine food and retained under normal conditions for $48 \mathrm{~h}$. Animals were anaesthetized to remove hairs from rear leg and wound $(3 \times 3 \mathrm{~cm})$ was made. The prepared dressing and commercial Band-aid ${ }^{\circledR}$ patch were then placed on the wounds in test and standard groups, respectively. However, wounds of control group were kept open. Advancement in wound healing was observed using tracing paper (Fig. 2). ${ }^{40}$

The samples of wound tissue of rat model preserved in $10 \%$ neutral buffer formalin were histopathologically examined. Briefly, before sectioning, tissue sample were embedded in paraffin. Later on with cryomicrotome (Leica RM 2145, Germany) tissue samples were cut into small sections $(4 \mu \mathrm{m})$ and stained by hematoxylin and eosin ( $\mathrm{H} \& \mathrm{E})$ staining. Following $\mathrm{H}$ \& E staining, the slides were observed for histological changes under an optical microscope (Olympus, BX-41). These studies

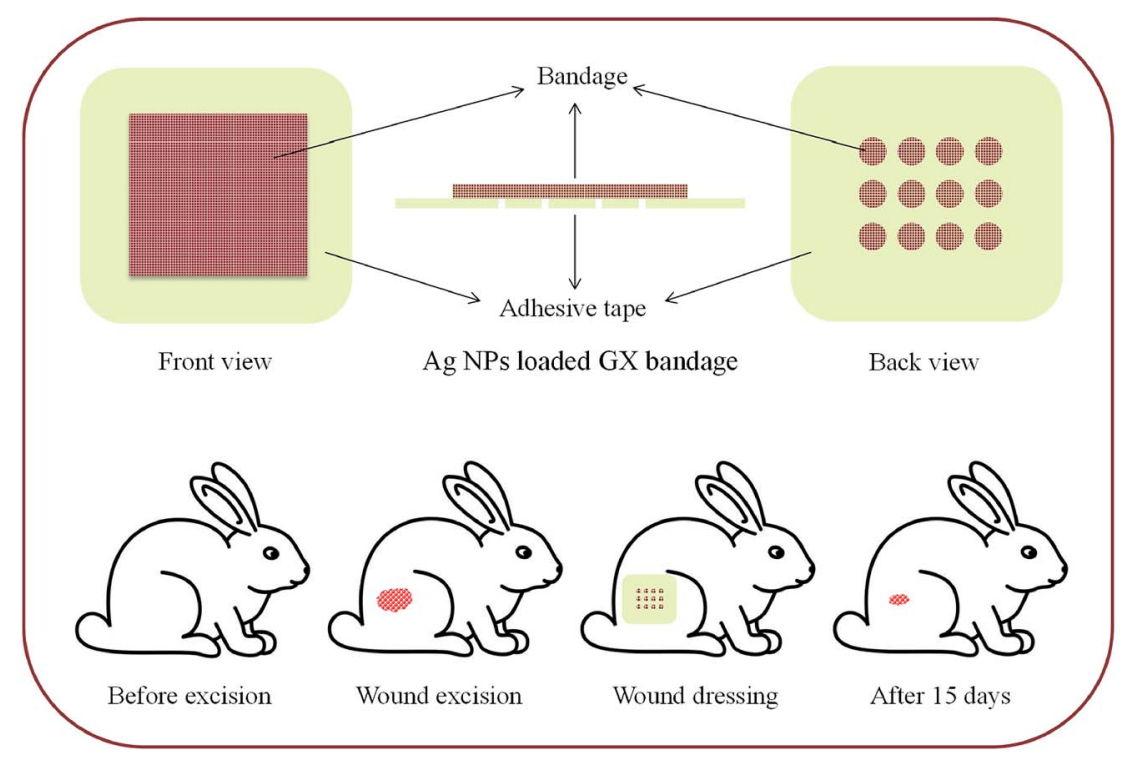

Fig. 2 Schematic diagram showing preparation of wound dressing and wound treatment. 
protocols was approved by Pharmacy Research Ethics Committee of University of Sargodha, Sargodha, Pakistan.

\subsection{Estimation of collagen}

For the evaluation of wound healing, collagen separated from wounds was suspended and stirred in acetic acid solution $(0.5 \mathrm{M}, 10 \% \mathrm{w} / \mathrm{v})$ for $48 \mathrm{~h}$ after washing with sodium acetate solution $(0.5 \mathrm{M})$. This solution was centrifuged at $5000 \mathrm{~g}$ for $2 \mathrm{~h}$. Collagen was precipitated using sodium chloride $(10 \% \mathrm{w} / \mathrm{v})$ solution and then filtered. ${ }^{37}$ The data obtained from the estimation of collagen were shown as the mean \pm S.D. and one-way ANOVA was used for analysis. $p$ value $<0.05$ was considered statistically significant and denoted by asterisk in the figure.

\subsection{Statistical analysis}

The data of antimicrobial study and collagen estimation were analyzed using one way ANOVA and values were expressed as mean \pm standard deviation. A $p<0.05$ was set as statistical significance.

\section{Results and discussion}

\subsection{Synthesis of Ag NPs}

Owing to biocompatible and ecofriendly nature, polysaccharides are widely used for the synthesis of NPs. ${ }^{23,24}$ The silver ions are reduced by accepting hydrated electrons from the solution after irradiating with sunlight. Actually, a complex $[\mathrm{Ag}(\mathrm{GX})]^{+}$is formed initially when solutions of silver nitrate and GX are homogenized. This complex is further reduced to $[\mathrm{Ag}(\mathrm{GX})]$, in which silver atoms tend to nucleate in form of NPs stabilized by the hydroxyl groups present on GX chains (Fig. 3a). All it is also evident from change in colour from colourless to reddish brown with respect to increase in exposure time of sample to sunlight (Fig. 3b).

\subsection{Characterization of Ag NPs}

3.2.1 UV/vis spectrophotometry. UV-visible spectrophotometry was used to investigate the synthesis of GX-Ag NPs from $\mathrm{AgNO}_{3}$ solutions (50, 75 and $100 \mathrm{mmol} ; 2 \mathrm{~mL}$ aliquot each) and GX hydrogel $(2 \mathrm{~mL})$. The colour of solution varies with time by reduction of $\mathrm{Ag}^{+}$to $\mathrm{Ag}$ NPs upon exposure to sunlight (Fig. 4). SPR phenomenon originated owing to combined oscillation of conducting electrons in Ag NPs help them to absorb strongly in the visible region. ${ }^{41}$ SPR transitions depend upon size and reaction time of $\mathrm{Ag}$ NPs which demonstrated colour changes from yellowish-brown to reddish-brown.

The characteristic SPR peaks of prepared GX-Ag NPs appeared in their UV-visible spectra at 390, 417, 438, 448 and $460 \mathrm{~nm}$ for $50 \mathrm{mmol}$; 397, 425, 443, 451 and $463 \mathrm{~nm}$ for $75 \mathrm{mmol}$; and 420, 440, 449, 459 and $465 \mathrm{~nm}$ for $100 \mathrm{mmol}$ $\mathrm{AgNO}_{3}$ solutions indicating the formation of NPs at respective reaction time $0.5,1,2,5$ and 24 h. The wavelength of absorbed light (bathochromic shift) increases with time due to increase in size of Ag NPs. Moreover, intensity of absorption also enhances with time from $30 \mathrm{~min}$ to $24 \mathrm{~h}$, indicating the constant reduction of $\mathrm{Ag}^{+}$ions by GX. Fig. 4 exhibits the UV-vis spectra of prepared GX-Ag NPs.

3.2.2 FT-IR spectroscopy. Hydroxyl groups present on GX chains surrounded Ag NPs to stabilize them. FT-IR spectra were obtained to confirm the interaction between - $\mathrm{OH}$ groups and $\mathrm{Ag}$ NPs (Fig. 5). FT-IR spectra showed that C-O-C stretching $\left(1047 \mathrm{~cm}^{-1}\right)$ of GX was shifted to a large band $\left(1109 \mathrm{~cm}^{-1}\right)$ for $\mathrm{Ag}$ NPs. Appearance of a new band at $464.8-592 \mathrm{~cm}^{-1}$ due to $\mathrm{Ag}-\mathrm{O}$ van der Waals forces confirms the formation of Ag NPs. ${ }^{19,20}$ Other absorption bands were found similar in pure GX and $\mathrm{Ag}$
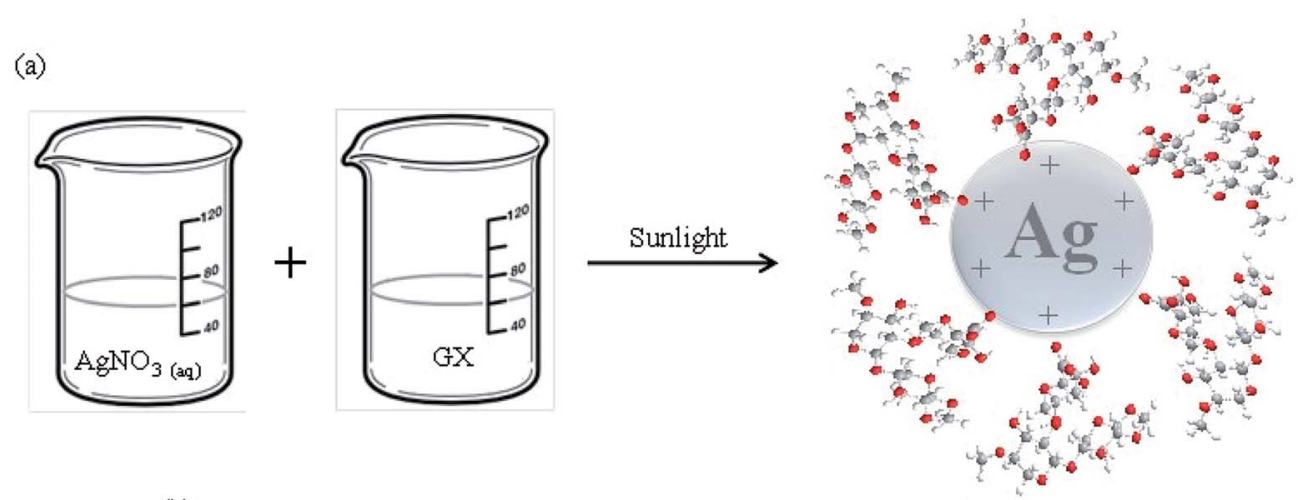

(b) Ag NP capped by GX

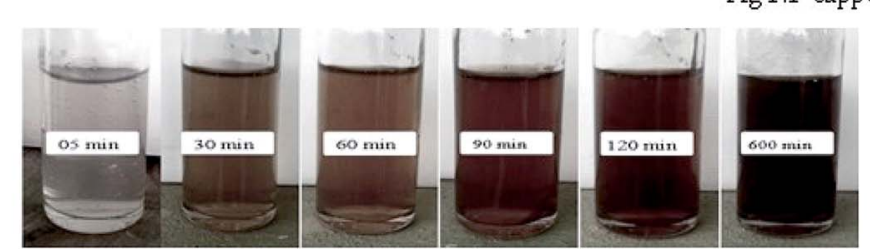

Fig. 3 Depiction of interactions between the hydroxyl groups of GX and positive charge of Ag NPs (a) and change in color on exposure to sunlight (b). 

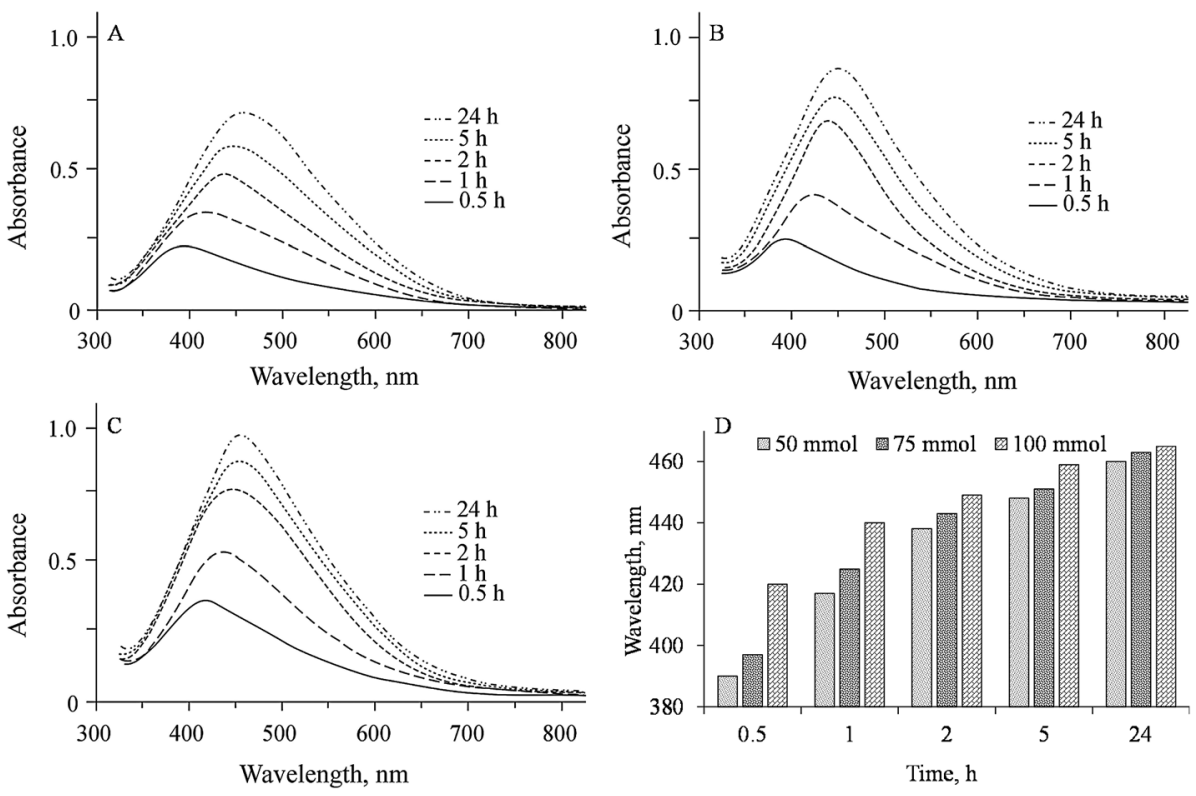

Fig. 4 UV/vis spectra of Ag NPs synthesized using GX with varying concentrations of $\mathrm{AgNO}_{3}$, i.e. $50 \mathrm{mmol}$ (A), $75 \mathrm{mmol}(\mathrm{B}), 100 \mathrm{mmol}(\mathrm{C})$ and combined graphical depiction exhibiting enhanced SPR absorption bands of solutions with time and concentration (D).

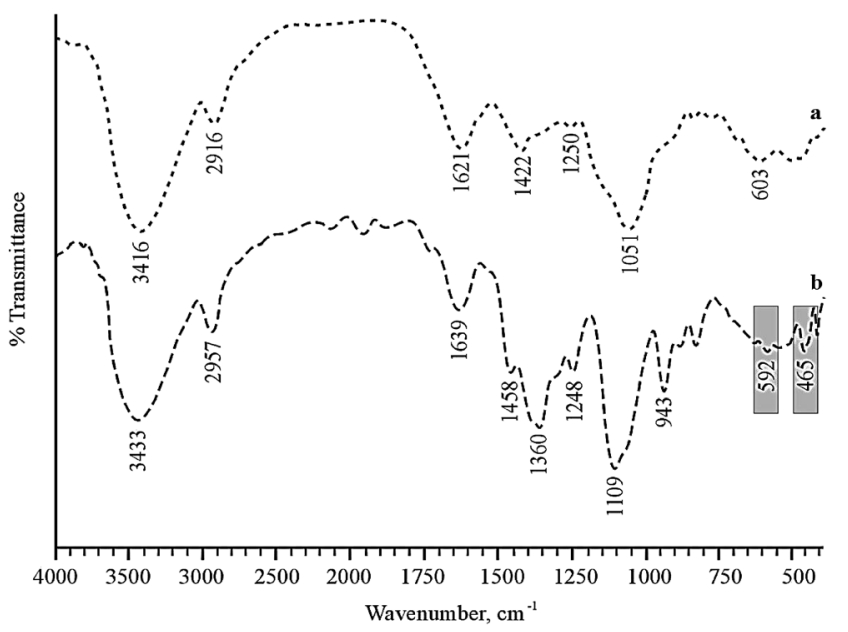

Fig. 5 FTIR spectra of GX and GX based Ag NPs.

NPs. Similar FT-IR spectra were recorded for $75 \mathrm{mmol}$ and 100 mmol solutions of Ag NPs.

3.2.3 SEM-EDS analyses. The size distribution of GX-Ag NPs confirmed using TEM mode of SEM, was found to broad in size range with uneven morphology, i.e., $9.3 \pm 5.7,10.6 \pm 3.7$ and $9.3 \pm 4.2 \mathrm{~nm}$ prepared separately from $2 \mathrm{~mL}$ of 50,75 and $100 \mathrm{mmol}$ solution of $\mathrm{AgNO}_{3}$ reduced/capped against $2 \mathrm{~mL}$ aqueous solution $(1 \% \mathrm{w} / \mathrm{v})$ of GX. The presence of silver in GXAg NPs was confirmed from their characteristic SPR absorption bands and additionally from EDS spectra as shown in Fig. 6 .

3.2.4 Powder X-ray diffraction. PXRD analyses were performed in order to confirm presence of crystal phase of the isolated $\mathrm{Ag}$ NPs in the range of $10-80^{\circ}, 2 \Theta$. The diffraction peaks centered at (111), (200), (220) and (311) indicated that Ag
NPs had face-centered cubic lattice in 50, 75 and $100 \mathrm{mmol}$ samples (Fig. 7), whereas QH showed highly amorphous nature.

\subsection{Storage of Ag NPs in GX thin films}

Potential of hydrogels based on polysaccharides for the longterm storage without any agglomeration of in situ synthesized $\mathrm{Ag}$ NPs has been evaluated in different studies. ${ }^{19,20}$ Therefore, GX based Ag NPs were evaluated for storage in the form of thin films. Thin films on drying under air and dark exhibited see through and foldable properties. Results of storage experiments are illustrated in Fig. 8. Ag NPs were synthesized by reducing the $\mathrm{AgNO}_{3}(50 \mathrm{mmol})$ solution with GX over period of $5 \mathrm{~h}$ then sample was stored as dry thin film under dark for further studies. Absorption spectra of the isolated Ag NPs were acquired by dissolving these thin films in deionized water each time. UV/ vis results have indicated that comparable spectra of Ag NPs were obtained after the storage period (12 and $24 \mathrm{~h}, 15$ and 30 days, 03 and 06 months) as no significant change in the absorption wavelength and intensity was observed for the stored samples. PXRD analysis of stored sample was also recorded after six months and found comparable to that of fresh samples. Hence, it is concluded that as synthesized Ag NPs on storage in thin films did not undergo any agglomeration.

\subsection{Antimicrobial activity of Ag NPs}

The as synthesized Ag NPs inhibited the growth of bacteria and fungi significantly. The zones of inhibition against $S$. epidermidis, S. aureus, P. aeruginosa, B. subtilis, E. coli, R. stolonifer, A. odontolyticus, $P$. notatum, and A. niger were found to be 16, 15, $24,13,21,15,16,14$ and $18 \mathrm{~mm}$ for $50 \mathrm{mmol}$; 14.5, 12.5, 21, 11, 18.5, 13, 13, 12 and 16 for $75 \mathrm{mmol}$; 14, 12, 21.5, 10, 18, 12, 14, 11 and 15 for $100 \mathrm{mmol}$ solutions of Ag NPs reduced the growth 

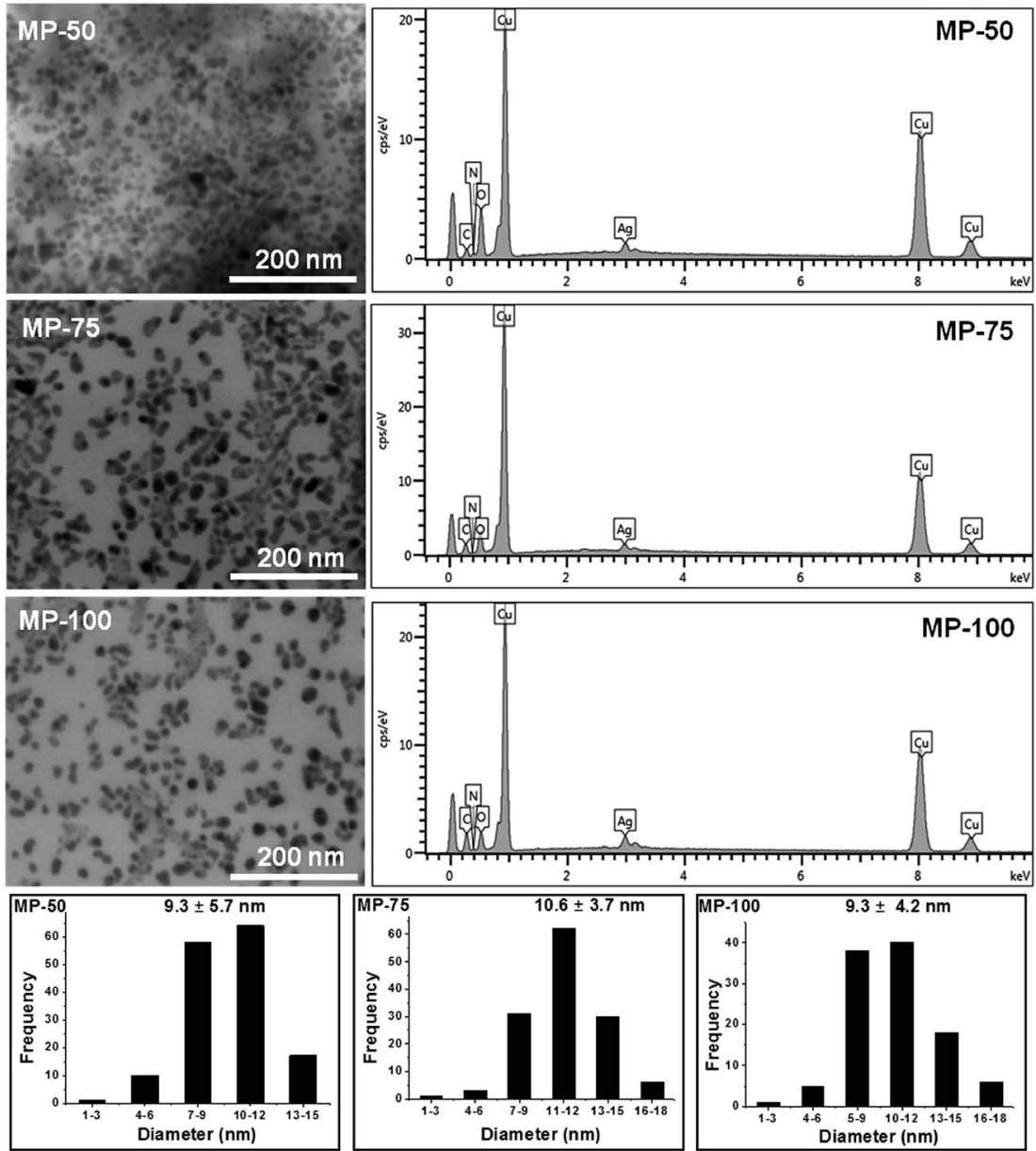

Fig. 6 TEM images along with their histograms and EDS of GX-Ag NPs produced by 50, 75 and 100 mmol concentration of AgNO 3

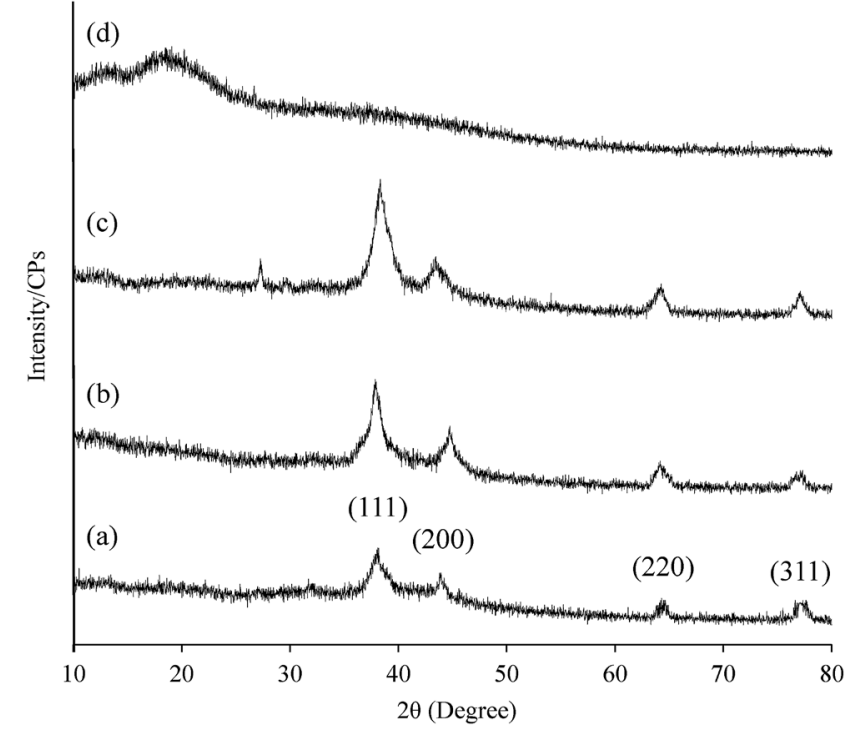

Fig. 7 PXRD spectra of isolated Ag NPs $50 \mathrm{mmol}$ (a), $75 \mathrm{mmol}$ (b) $100 \mathrm{mmol}(\mathrm{c})$ and GX (d). of microorganisms (Fig. 9) while GX and distilled water did not depict any antimicrobial activity. However, $\mathrm{AgNO}_{3}$ solution (0.01 M) displayed reasonable reduction in the growth of aforesaid strains. All the assays were performed thrice and the average values have been discussed.

The values of MIC of synthesized Ag NPs $(9.3 \pm 5.7 \mathrm{~nm})$, prepared with $50 \mathrm{mmol}$ solution of $\mathrm{AgNO}_{3}$, against different microbial strains $E$. coli and $S$. aureus were found in the range from 1.56 and $3.12 \mu \mathrm{g} \mathrm{mL}{ }^{-1}$, respectively. Increased surface area of smaller Ag NPs (9.3 nm, average size) are beneficial in attaching to the surface of bacterial cell wall and also having great tendency to penetrate the cell wall, hence exhibited high antimicrobial activity and less MIC value. ${ }^{42}$ Almost similar MIC value of such a small sized Ag NPs is reported by Fernandez et al., 2008 and Martinez et al., 2008. ${ }^{\mathbf{4 3 , 4 4}}$

\subsection{Wound healing studies}

For the evaluation of wound healing process using GX-Ag NPs, excision wound was created on rear leg of rabbit and average diameter $\left(\mathrm{cm}^{2}\right)$ of wounds was noted for different groups after fixed intervals. Among rabbits of untreated group, wound closure was observed to be $0.18,0.83,17.19$, $34.21,51.07$ and $77.41 \%$ on 1 st, 3rd, 6th, 9th, 12 th and 15 th 


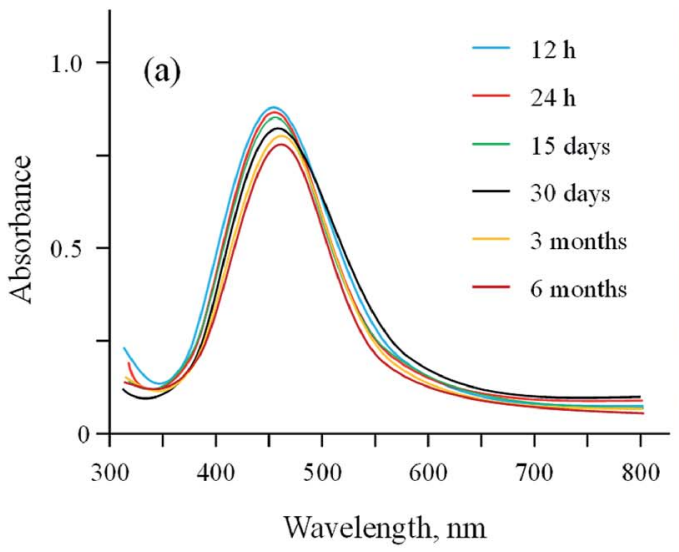

(111)

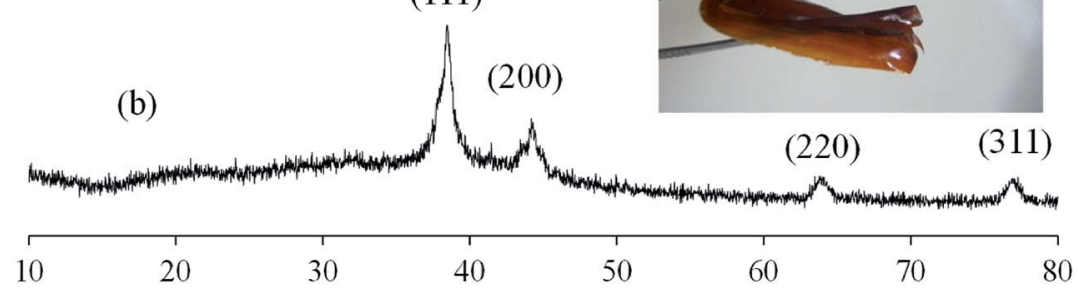

Fig. $8 \mathrm{UV} / \mathrm{vis}$ spectra of $\mathrm{AgNO}_{3}(50 \mathrm{mmol}$ ) solution in $\mathrm{GX}$ recorded after different storage times in film form (a), PXRD spectrum of Ag NPs isolated from GX-Ag NPs film after 6 months storage period (b), vial containing mixture of stored film in water (c), foldable and see through Ag NPs embedded GX thin film (d) and (e), respectively.

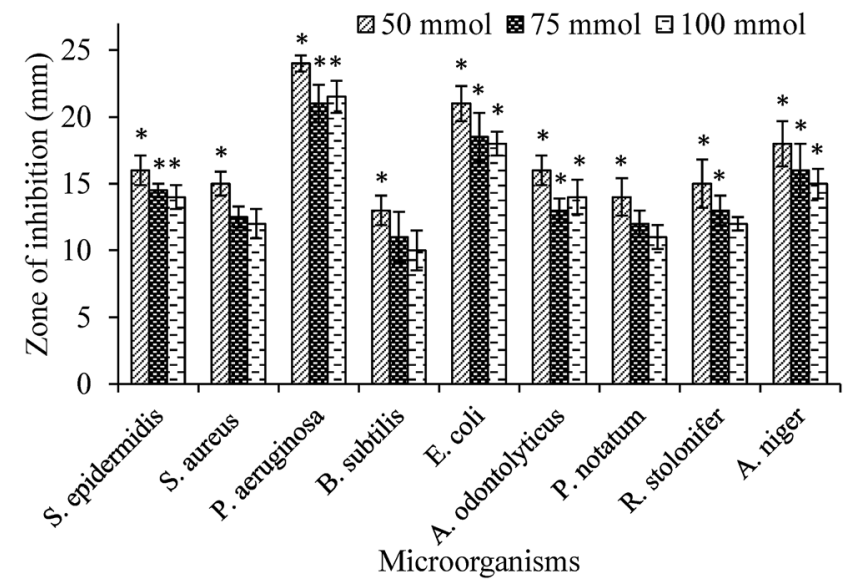

Fig. 9 Microbicidal effect of different concentrations of Ag NPs (50, 75 and $100 \mathrm{mmol})$. Statistical significance $(p<0.05)$ values from control are denoted by asterisks. days respectively while percentage wound closure in standard group treated with standard Band aid® dressing was 1.83, 21.54, 55.92, 81.67, 95.21 and 100\%. The GX-Ag NPs based dressings also displayed reasonable wound healing which was $1.13,15.24,61.29,83.74,97.34$ and $100 \%$ on $1 \mathrm{st}, 3 \mathrm{rd}, 6 \mathrm{th}$, 9th, 12th and 15th days, respectively. At the end of study, it was concluded that GX Ag NPs and Band aid® treated groups showed almost comparable wound healing capabilities. The percentage closure and diameter of wounds on different days are shown in Table 1. Hydrogel layer don't allow the cotton bandage to fix in the wound area (due to moisture) hence patient compliance can be achieved by developing such bandages.

The collagen content and tensile strength of the epithelium tissues were examined to understand mechanism of wounds. On 15th day, collagen contents for test group (74 $\left.\mathrm{mg} \mathrm{kg}^{-1}\right)$ were found higher than standard $\left(71 \mathrm{mg} \mathrm{kg}^{-1}\right)$ and control groups (42 $\mathrm{mg} \mathrm{kg}^{-1}$ ) undoubtedly revealing rapid curative process (Fig. 10). This rapid wound healing tendency was due to the

Table 1 Percentage wound healing along with diameter of wounds after fixed days in rabbit model

\begin{tabular}{|c|c|c|c|c|c|c|}
\hline \multirow[b]{2}{*}{ Day } & \multicolumn{6}{|c|}{ Diameter of wounds (cm \pm SD) with wound closure (\%) } \\
\hline & $1^{\text {st }}$ & $3^{\text {rd }}$ & $6^{\text {th }}$ & $9^{\text {th }}$ & $12^{\text {th }}$ & $15^{\text {th }}$ \\
\hline Control & $2.99 \pm 0.02(0.18)$ & $2.97 \pm 0.03(0.83)$ & $2.48 \pm 0.01(17.19)$ & $1.98 \pm 0.08(34.21)$ & $1.47 \pm 0.04(51.07)$ & $\begin{array}{l}1.35 \pm 0.04 \\
(77.41)\end{array}$ \\
\hline Band aid $\circledast$ dressing & $2.94 \pm 0.05(1.83)$ & $2.35 \pm 0.03(21.54)$ & $1.32 \pm 0.02(55.92)$ & $0.55 \pm 0.07(81.67)$ & $0.14 \pm 0.06(95.21)$ & $(100)$ \\
\hline GX-Ag NPs based dressing & $2.97 \pm 0.02(1.13)$ & $2.54 \pm 0.05(15.24)$ & $1.16 \pm 0.02(61.29)$ & $0.49 \pm 0.09(83.74)$ & $0.08 \pm 0.02(97.34)$ & $(100)$ \\
\hline
\end{tabular}




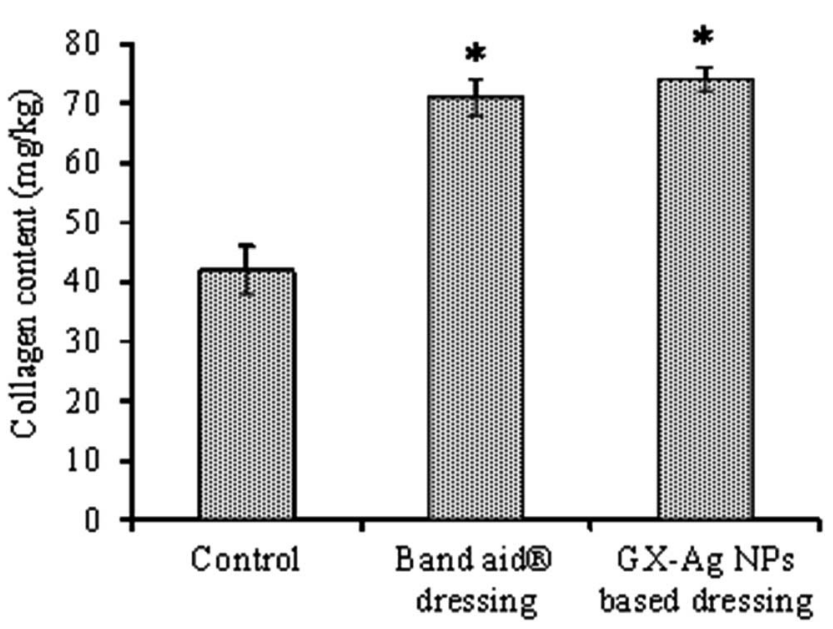

Fig. 10 The contents of collagen at wounds of various groups on 15th day. Statistical significance difference $(p<0.05)$ from control is represented by asterisks.

crosslinking of collagen fibers. Furthermore, the tensile strength of epithelium tissues in test group was also greater than standard and control groups. In reality, collagen begins establishing at wounds when polypeptide precursor is produced.

Histological examination of wounded tissues of rats on day 7 and 15 is illustrated in Fig. 11. This study of the wound healing revealed that at day 7 , in all groups epidermis was not well developed and was covered by acute inflammatory cells. The presence of circulating inflammatory cells such as neutrophils was evident and re-epithelization took place on the wound edge. Regeneration of new epithelium appeared on day 15. On day 15 GX-Ag NPs $(50 \mathrm{mM})$ and Band aid ${ }^{\circledR}$ treated group showed the highest rate of re-epithelialization. Moreover on day 15, epithelialization was nearly complete and GX-Ag NPs and Band aid $®$ treated group showed a thick and organized epidermis, an apparent re-epithelialization and well-formed granulation tissue was evident.

\section{Conclusions}

In present investigation, we introduced an economical and green method for the synthesis of Ag NPs based on a naturally occurring glucuronoxylan (GX) polysaccharide. The role of GX polysaccharide in for formation and stabilization and storage was thoroughly evaluated. The surface morphology and elemental composition of prepared NPs have been evaluated using SEM equipped with STEM and EDS modes. The obtained small sized nanoparticles with definite geometry may show valuable applications in many fields as in present work GX based Ag NPs were found to exhibit significant microbicidal potential. The stability of the Ag NPs was achieved up to six months storage in the form of thin films based on in situ present GX. Likewise, GX-Ag NPs based dressing showed great potential for rapid wound healing applications due to high microbicidal properties of small sized Ag NPs present in the bandage. The as-synthesized NPs may find their applications and surface plasmon resonance based biomedical diagnostics and catalysis. Besides these applications of GX-Ag NPs studied herein, such Ag NPs-glucuronoxylan composite material may show great potential applications in catalysis, therapeutics, nanobiotechnology, wound management, pharmaceuticals and daily life.

\section{Conflicts of interest}

There are no conflicts of interest to declare.

\section{Acknowledgements}

G. Muhammad gratefully acknowledges the Higher Education Commission, Pakistan, for funding under the scheme "HEC Indigenous 5000 Fellowships".
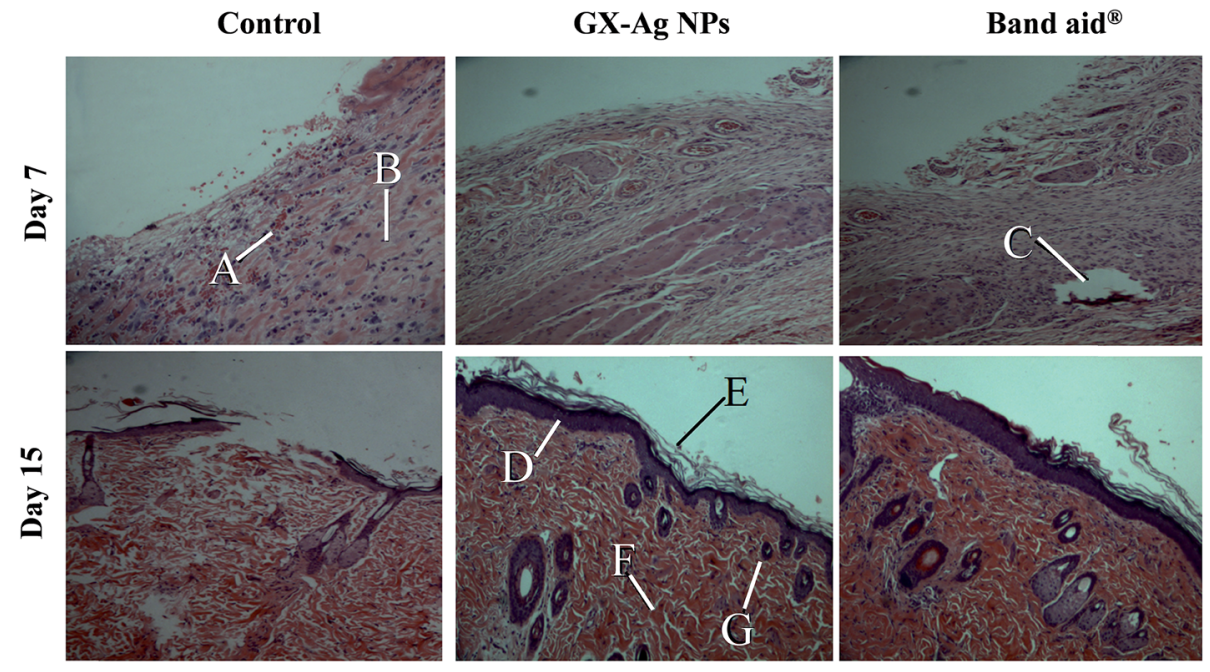

Fig. 11 Histological examination of wound healing on day 7, and 15 by H \& E staining. (A) Neutrophils accumulation; (B) collagen deposition; (C) hair follicle; (D) epidermis; (E); stratum corneum, (F) dermis and (G) fibroblasts. 


\section{References}

1 A. Nel, T. Xia, L. Madler and N. Li, Science, 2006, 311, 622627.

2 C. J. Murphy, Science, 2002, 298, 2139-2141.

3 B. Nowack, H. F. Krugm and M. Height, Environ. Sci. Technol., 2011, 45, 1177-1183.

4 M. Ahamed, M. S. AlSalhi and M. K. J. Siddiqui, Clin. Chim. Acta, 2010, 411, 1841-1848.

5 X. Chen and H. J. Schluesener, Toxicol. Lett., 2008, 176, 1-12.

6 D. P. O'Neal, L. R. Hirsch, N. J. Halas, J. D. Payne and J. L. West, Cancer Lett., 2004, 209, 171-176.

7 I. Pastroiza-Santos and L. M. Liz-Marzan, Langmuir, 2002, 18, 2888-2894.

8 M. Chen, L. Y. Wang, J. T. Han, J. Y. Zhang, Z. Y. Li and D. J. Qian, J. Phys. Chem. B, 2006, 110, 11224-11231.

9 M. Popa, T. Pradell, D. Crespo and J. M. Calderón-Moreno, Colloids Surf., A, 2007, 303, 184-190.

10 K. Ma, L. Gong, X. Cai, P. Huang, J. Cai, D. Huang and T. Jiang, Int. J. Nanomed., 2017, 12, 3665-3679.

11 S. Huang, Z. Yu, Y. Zhang, C. Qi and S. Zhang, Int. J. Nanomed., 2017, 12, 3181-3191.

12 M. M. Husein, E. Rodil and J. H. Vera, J. Colloid Interface Sci., 2005, 288, 457-467.

13 N. M. Huang, S. Radiman, H. N. Lim, P. S. Khiew, W. S. Chiu, K. H. Lee, A. Syahida, R. Hashim and C. H. Chia, Chem. Eng. J., 2009, 155, 499-507.

14 M. Darroudi, M. B. Ahmad, K. Shameli, A. H. Abdullah and N. A. Ibrahim, Solid State Sci., 2009, 17, 1621-1624.

$15 \mathrm{H}$. Yin, T. Yamamoto, Y. Wada and S. Yanagida, Mater. Chem. Phys., 2004, 83, 66-70.

16 H. S. Kim, K. H. Lee and S. G. Kim, Aerosol Sci. Technol., 2006, 40, 536-544.

17 K. Vimala, K. S. Sivudu, Y. M. Mohan, B. Sreedhar and K. M. Raju, Carbohydr. Polym., 2009, 75, 463-471.

18 G. F. Prozorova, A. S. Pozdnyakov, N. P. Kuznetsova, S. A. Korzhova, A. I. Emel'yanov, T. G. Ermakova, T. V. Fadeeva and L. M. Sosedova, Int. J. Nanomed., 2014, 9, 1883-1889.

19 M. A. Hussain, A. Shah, I. Jantan, M. R. Shah, M. N. Tahir, R. Ahmad and S. N. A. Bukhari, Int. J. Nanomed., 2015, 10, 2079-2088.

20 M. A. Hussain, A. Shah, I. Jantan, M. N. Tahir, M. R. Shah, R. Ahmad and S. N. A. Bukhari, J. Nanobiotechnol., 2014, 12, 53 .

21 J. Cai, S. Kimura, M. Wada and S. Kuga, Biomacromolecules, 2009, 10, 87-94.

22 G. R. Barbari, F. A. Dorkoosh, M. Amini, M. Sharifzadeh, F. Atyabi, S. Balalaei, N. R. Tehrani and M. R. Tehrani, Int. J. Nanomed., 2017, 12, 3471-3483.

23 M. Amin, F. Anwar, M. R. S. A. Janjua, M. A. Iqbal and U. Rashid, Int. J. Mol. Sci., 2012, 13, 9923-9941.
24 M. T. Haseeb, M. A. Hussain, K. Abbas, B. G. M. Youssif, S. Bashir, S. H. Yuk and S. N. A. Bukhari, Int. J. Nanomed., 2017, 12, 2845.

25 A. S. Hoffman, Adv. Drug Delivery Rev., 2012, 64, 18-23.

26 M. A. Hussain, G. Muhammad, I. Jantan and S. N. A. Bukhari, Polym. Rev., 2016, 56, 1-30.

27 B. T. Good, C. N. Bowman and R. H. Davis, Chem. Eng. Sci., 2004, 59, 5967-5974.

28 G. Muhammad, M. A. Hussain, I. Jantan and S. N. A. Bukhari, Compr. Rev. Food Sci. Food Saf., 2015, 15, 303-315.

29 G. Muhammad, M. A. Hussain, M. U. Ashraf, M. T. Haseeb, S. Z. Hussain and I. Hussain, RSC Adv., 2016, 6, 2331023317.

30 M. U. Ashraf, M. A. Hussain, G. Muhammad, M. T. Haseeb, S. Bashir, S. Z. Hussain and I. Hussain, Int. J. Biol. Macromol., 2017, 95, 138-144.

31 M. Ahuja, A. Kumar, P. Yadav and K. Singh, Int. J. Biol. Macromol., 2013, 57, 105-110.

32 V. Kumar and S. Kumar, Int. J. Pharm. Pharm. Sci., 2011, 3, 55-57.

33 K. Singh, A. Kumar, L. Langyan and M. Ahuja, AAPS PharmSciTech, 2009, 10, 1121-1127.

34 F. Iram, M. S. Iqbal, M. M. Athar, M. Z. Saeed, A. Yasmeen and R. Ahmad, Carbohydr. Polym., 2014, 104, 29-33.

35 A. W. Bauer, W. M. Kirby, J. C. Sherris and M. Turck, Am. J. Clin. Pathol., 1966, 45, 493-496.

36 I. Wiegand, K. Hilpert and R. E. W. Hancock, Nat. Protoc., 2008, 3, 163-175.

37 M. Elshikh, S. Ahmed, S. Funston, P. Dunlop, M. McGaw, R. Marchant and I. M. Banat, Biotechnol. Lett., 2016, 38, 1015-1019.

38 S. D. Sarker, L. Nahar and Y. Kumarasamy, Methods, 2007, 42, 321-324.

39 Directive EU, 63/EU of the European parliament and of the council of 22 September 2010 on the protection of animals used for scientific purposes, Official Journal of the European Union, 2010, 276, 33-74.

40 K. H. Lee and T. G. Tong, J. Pharm. Sci., 1968, 57, 1042-1046. 41 S. Ashraf, A. Z. Abbasi, C. Pfeiffer, S. Z. Hussain, Z. M. Khalid, P. R. Gil, W. J. Parak and I. Hussain, Colloids Surf., B, 2013, 102, 511-518.

42 C. Marambio-Jones and E. M. V. Hoek, J. Nanopart. Res., 2010, 12, 1531-1551.

43 G. A. Martinez-Castanon, N. Nino-Martinez, F. MartinezGutierrez, J. R. Martinez-Mendoza and F. Ruiz, J. Nanopart. Res., 2008, 10, 1343-1348.

44 E. J. Fernandez, J. Garcia-Barrasa, A. Laguna, J. M. Lopez-de Luzuriaga, M. Monge and C. Torres, Nanotechnology, 2008, 19, 1-6. 